\title{
A New Approach for Linear Eigenvalue Problems and Nonlinear Euler Buckling Problem
}

\author{
Meltem Evrenosoglu Adiyaman and Sennur Somali \\ Department of Mathematics, Faculty of Science, Dokuz Eylul University, 35160 Tinaztepe, \\ Buca, Izmir, Turkey \\ Correspondence should be addressed to Meltem Evrenosoglu Adiyaman, \\ meltem.evrenosoglu@deu.edu.tr
}

Received 12 March 2012; Accepted 11 April 2012

Academic Editor: Allaberen Ashyralyev

Copyright (C) 2012 M. E. Adiyaman and S. Somali. This is an open access article distributed under the Creative Commons Attribution License, which permits unrestricted use, distribution, and reproduction in any medium, provided the original work is properly cited.

We propose a numerical Taylor's Decomposition method to compute approximate eigenvalues and eigenfunctions for regular Sturm-Liouville eigenvalue problem and nonlinear Euler buckling problem very accurately for relatively large step sizes. For regular Sturm-Liouville problem, the technique is illustrated with three examples and the numerical results show that the approximate eigenvalues are obtained with high-order accuracy without using any correction, and they are compared with the results of other methods. The numerical results of Euler Buckling problem are compared with theoretical aspects, and it is seen that they agree with each other.

\section{Introduction}

We investigate the computation of eigenvalues of regular Sturm-Liouville eigenvalue problems:

$$
\begin{gathered}
-y^{\prime \prime}(x)+r(x) y(x)=\lambda y(x), \quad 0 \leq x_{0}<x<x_{n}, \\
y\left(x_{0}\right)=y\left(x_{n}\right)=0,
\end{gathered}
$$

where $r(x) \in C^{p+q}\left[x_{0}, x_{n}\right]$ and $p, q \in \mathbb{N}$ and Euler Buckling problem:

$$
\begin{gathered}
y^{\prime \prime}+\lambda \sin y=0, \\
y^{\prime}(0)=0, \quad y^{\prime}(1)=0 .
\end{gathered}
$$

Regular Sturm-Liouville problems arise in many applications, and many methods are available for their numerical solution Pryce [1]. 
We also examine an elementary, classical problem buckling of an end-loaded rod which possesses a completely soluble continuous model in the form of a nonlinear, secondorder boundary value problem as described in elsewhere [2-5]. An essential complete analysis of this problem was provided by Euler [6]. For the nonlinear eigenvalue problem (1.2), one may find that for small $\lambda$ the only solution is zero solution as in the linear case. But as the eigenvalue $\lambda$ increases, it reaches a critical value $\lambda_{1}$ at which a nonzero solution appears, corresponding to buckling of the rod. For $\lambda>\lambda_{1}$, the nonlinear problem behaves quite differently from the linear problem: for a range of values $\lambda_{1}<\lambda<\lambda_{2}$, there is exactly one nonzero solution of (1.2) for each $\lambda$, and when $\lambda$ exceeds $\lambda_{2}$, a second nonzero solution appears; similarly, there is a value $\lambda_{3}$ beyond which there are three nonzero solutions, and so on. Namely, one may give inductively

$$
\begin{gathered}
0 \leq \lambda \leq \pi^{2}, \quad \text { only the trivial solution, } \\
\pi^{2}<\lambda \leq 4 \pi^{2}, \quad \text { one nontrivial solution, } \\
n^{2} \pi^{2}<\lambda \leq(n+1)^{2} \pi^{2}, \quad n \text { nontrivial solutions, }
\end{gathered}
$$

as given by Stakgold [2]. This behavior is a simple example of the phenomenon of bifurcation or branching; it occurs in many different areas of applied mathematics.

The method considered here is a Taylor decomposition which was used by Adiyaman and Somali [7] for the solution of certain nonlinear problems. Like classical finite-difference and finite-element methods, this high order method is best suited to the fundamental eigenvalue and small eigenvalues.

In Section 2, the behavior of eigenvalues and corresponding eigenfunctions for regular Sturm-Liouville problem is obtained by Taylor's decomposition method, and convergence of the method for regular Sturm-Liouville problem with constant function $r(x)$ is given. We establish a Lemma and a Theorem, and then we give an application of Taylor's decomposition method to the Euler Buckling problem in Section 3. The technique is illustrated with three examples, and the numerical results of regular Sturm-Liouville problem are given by comparing the results of other methods in Section 4 . The numerical results of Euler Buckling problem accompanying the theoretical results and the behavior of solution are also discussed in Section 4 . In the conclusion, we summarize the study and present our suggestions regarding future work.

\section{Application and Error Analysis of Taylor's Decomposition Method for Regular Sturm-Liouville Eigenvalue Problems}

\subsection{Application of Taylor's Decomposition on Two Points for Regular Sturm-Liouville Eigenvalue Problems}

We consider the regular Sturm-Liouville eigenvalue problem (1.1) by introducing the new depending variable $y^{\prime}(x)=z(x)$, (1.1) can be written as

$$
\begin{gathered}
Y^{\prime}(x)=A(x) Y(x), \\
C_{0} Y\left(x_{0}\right)+C_{1} Y\left(x_{n}\right)=0,
\end{gathered}
$$


where

$$
Y(x)=\left[\begin{array}{l}
y(x) \\
z(x)
\end{array}\right], \quad A(x)=\left[\begin{array}{cc}
0 & 1 \\
r(x)-\lambda & 0
\end{array}\right], \quad C_{0}=\left[\begin{array}{ll}
1 & 0 \\
0 & 0
\end{array}\right], \quad C_{1}=\left[\begin{array}{ll}
0 & 0 \\
1 & 0
\end{array}\right]
$$

From Ashyralyev and Sobolevskii [8], we will consider the application of Taylor's decomposition of function $Y(x)$ on two points. We need to find $Y^{(j)}(x)$ for any $1 \leq j \leq p$ and $q$. Using the equation $Y^{\prime}(x)=A(x) Y(x)$, we get

$$
Y^{(j)}(x)=A_{j}(x) Y(x)
$$

with

$$
\begin{gathered}
A_{0}(x)=I, \\
A_{1}(x)=A(x), \\
A_{j}(x)=A_{j-1}^{\prime}(x)+A_{j-1}(x) A(x), \quad 2 \leq j \leq p,
\end{gathered}
$$

where $I$ is the $2 \times 2$ identity matrix. By using the structure of the matrix $A(x)$, we obtain the entries of the matrix of

$$
A_{j}(x)=\left[\begin{array}{ll}
a_{j(1,1)}(\lambda ; x) & a_{j(1,2)}(\lambda ; x) \\
a_{j(2,1)}(\lambda ; x) & a_{j(2,2)}(\lambda ; x)
\end{array}\right]
$$

as in the following formulas:

$$
\begin{gathered}
a_{j(1,1)}(\lambda ; x)=\frac{\partial a_{j-1(1,1)}(\lambda ; x)}{\partial x}+(r(x)-\lambda) a_{j-2(2,2)}(\lambda ; x)=a_{j-1(2,1)}(\lambda ; x), \\
a_{j(2,2)}(\lambda ; x)=\frac{\partial a_{j-1(2,2)}(\lambda ; x)}{\partial x}+a_{j(1,1)}(\lambda ; x), \\
a_{j(1,2)}(\lambda ; x)=a_{j-1(2,2)}(\lambda ; x), \\
a_{j(2,1)}(\lambda ; x)=-\frac{\partial a_{j(2,2)}(\lambda ; x)}{\partial x}+a_{j+1(2,2)}(\lambda ; x),
\end{gathered}
$$

for $2 \leq j \leq p$, where

$$
\begin{array}{cc}
a_{0(1,1)}(\lambda ; x)=1, & a_{1(1,1)}(\lambda ; x)=0, \\
a_{0(1,2)}(\lambda ; x)=0, & a_{1(1,2)}(\lambda ; x)=1, \\
a_{0(2,1)}(\lambda ; x)=0, & a_{1(2,1)}(\lambda ; x)=r(x)-\lambda, \\
a_{0(2,2)}(\lambda ; x)=1, & a_{1(2,2)}(\lambda ; x)=0 .
\end{array}
$$


From the theorem given in Ashyralyev and Sobolevskii [8], we have the following relation:

$$
\begin{array}{r}
Y\left(x_{k}\right)-Y\left(x_{k-1}\right)+\sum_{j=1}^{p} \alpha_{j} Y^{(j)}\left(x_{k}\right) h^{j}-\sum_{j=1}^{q} \beta_{j} Y^{(j)}\left(x_{k-1}\right) h^{j} \\
=\frac{(-1)^{p}}{(p+q) !} \int_{x_{k-1}}^{x_{k}}\left(x_{k}-s\right)^{q}\left(s-x_{k-1}\right)^{p} Y^{(p+q+1)}(s) d s
\end{array}
$$

on the uniform grid

$$
\left[x_{0}, x_{n}\right]_{h}=\left\{x_{k}=x_{0}+k h, k=0,1, \ldots, n, n h=x_{n}-x_{0}, n \in N\right\},
$$

where

$$
\begin{gathered}
\alpha_{j}=\frac{(p+q-j) ! p !(-1)^{j}}{(p+q) ! j !(p-j) !}, \quad 1 \leq j \leq p \\
\beta_{j}=\frac{(p+q-j) ! q !}{(p+q) ! j !(q-j) !}, \quad 1 \leq j \leq q .
\end{gathered}
$$

Rewriting (2.8) by neglecting the last term, we obtain the single-step difference scheme of $(p+q)$-order of accuracy for the approximate solution of problem (2.1):

$$
Y_{k}-Y_{k-1}+\sum_{j=1}^{p} \alpha_{j} A_{j}\left(x_{k}\right) Y_{k} h^{j}-\sum_{j=1}^{q} \beta_{j} A_{j}\left(x_{k-1}\right) Y_{k-1} h^{j}=0,
$$

where

$$
Y_{k}=\left[\begin{array}{l}
y_{k} \\
z_{k}
\end{array}\right]
$$

is the approximate value of $Y\left(x_{k}\right)$. For the simple computation, let $p=q$, then we have

$$
\left(I+\sum_{j=1}^{p} \alpha_{j} A_{j}\left(x_{k}\right) h^{j}\right) Y_{k}=\left(I+\sum_{j=1}^{p}(-1)^{j} \alpha_{j} A_{j}\left(x_{k-1}\right) h^{j}\right) Y_{k-1}
$$

where $\beta_{j}=(-1)^{j} \alpha_{j}$. Letting $M\left(x_{k}\right)=\left(I+\sum_{j=1}^{p} \alpha_{j} A_{j}\left(x_{k}\right) h^{j}\right)$ and $N\left(x_{k-1}\right)=(I+$ $\left.\sum_{j=1}^{p}(-1)^{j} \alpha_{j} A_{j}\left(x_{k-1}\right) h^{j}\right)$, we write

$$
Y_{k}=M^{-1}\left(x_{k}\right) N\left(x_{k-1}\right) Y_{k-1}
$$


Since the accuracy and convergence of the method not only depend on $h$, they also depend on $p$, we can increase the order of accuracy by increasing $p$ for fixed $h$. So $h$ is chosen as length of the whole interval as follows. Now, taking $h=x_{n}-x_{0}$ gives

$$
Y_{1}=M^{-1}\left(x_{n}\right) N\left(x_{0}\right) Y_{0}
$$

and substituting into the boundary condition of (2.1), we get

$$
\left(C_{1} M^{-1}\left(x_{n}\right) N\left(x_{0}\right)+C_{0}\right) Y_{0}=0
$$

To obtain a nontrivial solution $Y_{0}$, we must have the following equation:

$$
\operatorname{det}\left(C_{1} M^{-1}\left(x_{n}\right) N\left(x_{0}\right)+C_{0}\right)=0 .
$$

Defining

$$
M\left(x_{n}\right)=\left[\begin{array}{ll}
m_{11} & m_{12} \\
m_{21} & m_{22}
\end{array}\right], \quad N\left(x_{0}\right)=\left[\begin{array}{ll}
n_{11} & n_{12} \\
n_{21} & n_{22}
\end{array}\right],
$$

we have the following statement

$$
m_{22} n_{12}-m_{12} n_{22}=0 .
$$

Since

$$
\begin{gathered}
M\left(x_{n}\right)=\left[\begin{array}{cc}
1+\sum_{j=1}^{p} \alpha_{j} a_{j(1,1)}\left(\lambda ; x_{n}\right) h^{j} & \sum_{j=1}^{p} \alpha_{j} a_{j(1,2)}\left(\lambda ; x_{n}\right) h^{j} \\
\sum_{j=1}^{p} \alpha_{j} a_{j(2,1)}\left(\lambda ; x_{n}\right) h^{j} & 1+\sum_{j=1}^{p} \alpha_{j} a_{j(2,2)}\left(\lambda ; x_{n}\right) h^{j}
\end{array}\right], \\
N\left(x_{0}\right)=\left[\begin{array}{cc}
1+\sum_{j=1}^{p}(-1)^{j} \alpha_{j} a_{j(1,1)}\left(\lambda ; x_{0}\right) h^{j} & \sum_{j=1}^{p}(-1)^{j} \alpha_{j} a_{j(1,2)}\left(\lambda ; x_{0}\right) h^{j} \\
\sum_{j=1}^{p}(-1)^{j} \alpha_{j} a_{j(2,1)}\left(\lambda ; x_{0}\right) h^{j} & 1+\sum_{j=1}^{p}(-1)^{j} \alpha_{j} a_{j(2,2)}\left(\lambda ; x_{0}\right) h^{j}
\end{array}\right],
\end{gathered}
$$


using the entries $m_{12}, m_{22}, n_{12}$, and $n_{22}$ of the above matrices and the properties of the entries of $A_{j}(x)$, we obtain (2.19) in terms of $\lambda$ :

$$
\begin{aligned}
F(\lambda)= & m_{22} n_{12}-m_{12} n_{22} \\
= & \left(1+\sum_{j=1}^{p} \alpha_{j} a_{j(2,2)}\left(\lambda ; x_{n}\right) h^{j}\right)\left(\sum_{j=1}^{p}(-1)^{j} \alpha_{j} a_{j-1(2,2)}\left(\lambda ; x_{0}\right) h^{j}\right) \\
& -\left(\sum_{j=1}^{p} \alpha_{j} a_{j-1(2,2)}\left(\lambda ; x_{n}\right) h^{j}\right)\left(1+\sum_{j=1}^{p}(-1)^{j} \alpha_{j} a_{j(2,2)}\left(\lambda ; x_{0}\right) h^{j}\right)
\end{aligned}
$$

Solving nonlinear equation $F(\lambda)=0$ by Newton's method, we find the approximate eigenvalues. This method appears to require a separate calculation for the eigenfunctions.

To find the corresponding eigenfunctions of the regular Sturm-Liouville eigenvalue problem (2.1), we substitute the eigenvalue to (2.1) and we solve the obtained boundary value problem by Taylor's decomposition method on two points $x_{k-1}$ and $x_{k}$ with the uniform grid $[0,1]_{h}$ for $p=q$. Then, we get a homogeneous linear equation system of $2 n$ equations with $2 n$ unknown $z_{0}, y_{1}, z_{1}, y_{2}, z_{2} \ldots, y_{n-1}, z_{n-1}, z_{n}$ which are the approximated values of $y^{\prime}\left(x_{0}\right), y\left(x_{1}\right), y^{\prime}\left(x_{1}\right), y\left(x_{2}\right), y^{\prime}\left(x_{2}\right), \ldots, y\left(x_{n-1}\right), y^{\prime}\left(x_{n-1}\right), y^{\prime}\left(x_{n}\right)$, respectively. Solving the $2 n \times 2 n$ homogeneous system, we obtain approximate values of the eigenfunction and its derivative of (1.1) at the point $x=x_{k}$.

\subsection{Error Analysis for Regular Sturm-Liouville Problem When $r(x)=c$}

In this section, we will show the convergence of the method for eigenfunctions with the constant function $r(x)=c$ by obtaining approximate value of eigenfunction at the point $\bar{x} \in\left[x_{0}, x_{n}\right]$ of the problem (1.1). Without loss of generality, we may choose $r(x)=0$, then $A_{j}(x)=A_{j}$, that is, $a_{j(2,2)}\left(\lambda ; x_{n}\right)=a_{j(2,2)}(\lambda ; 0)=a_{j(2,2)}(\lambda)$. Using $(2.6)$, we can find explicit values of $a_{j(1,1)}, a_{j(2,2)}$ as follows:

$$
\begin{gathered}
a_{2 j(1,1)}=(-1)^{j} \mathcal{\lambda}^{j}, \\
a_{2 j(2,2)}=(-1)^{j} \mathcal{\lambda}^{j}, \\
a_{2 j+1(1,1)}=0, \\
a_{2 j+1(2,2)}=0, \quad j \geq 0 .
\end{gathered}
$$


This yields

$$
\begin{gathered}
m_{22}=1+\sum_{j=1}^{\lfloor p / 2\rfloor} \alpha_{2 j}(-1)^{j} \lambda^{j} h^{2 j}, \\
n_{22}=m_{22}, \\
m_{12}=\sum_{j=0}^{\lfloor(p-1) / 2\rfloor} \alpha_{2 j+1}(-1)^{j} \lambda^{j} h^{2 j+1}, \\
n_{12}=-m_{12}, \\
m_{11}=m_{22}, \\
m_{21}=-\lambda m_{12} .
\end{gathered}
$$

Using (2.14) for $k=1$, we have

$$
Y_{1}=M^{-1}(\bar{x}) N\left(x_{0}\right) Y_{0}
$$

where $Y_{0}$ and $Y_{1}$ are the approximated values of $Y\left(x_{0}\right)$ and $Y(\bar{x})$, respectively, with the stepsize $h=\bar{x}-x_{0}$ :

$$
Y_{1}=\frac{z_{0}}{\operatorname{det}(M)}\left[\begin{array}{c}
-2 m_{22} m_{12} \\
-\lambda\left(m_{12}\right)^{2}+\left(m_{22}\right)^{2}
\end{array}\right]
$$

The first component of the above vector (2.25) gives the approximate eigenfunction $y_{1}$, and the second component of the above vector (2.25) gives the derivative of the approximate eigenfunction $z_{1}$ of the regular Sturm-Liouville problem (1.1) at $\bar{x}$. Now, we will show that $y_{1}$ and $z_{1}$ converge to exact functions $y(\bar{x})$ and $y^{\prime}(\bar{x})$, respectively, as $p \rightarrow \infty$.

Using the Stirling's Formula $n ! \approx \sqrt{2 \pi} n^{(n+1) / 2} e^{-n}$ for $\alpha_{j}$ in (2.10), we obtain

$$
\alpha_{j}=\frac{(2 p-j) ! p !(-1)^{j}}{(2 p) ! j !(p-j) !} \approx(-1)^{j} \frac{1}{j !} \frac{1}{2^{j}}\left(\frac{p-j / 2}{p-j}\right)^{(p-j+1) / 2}\left(\frac{p-j / 2}{p}\right)^{p / 2} .
$$

This gives

$$
\lim _{p \rightarrow \infty} \alpha_{j}=(-1)^{j} \frac{1}{j !} \frac{1}{2^{j}} .
$$


Thus,

$$
\begin{aligned}
\lim _{p \rightarrow \infty} m_{22} & =\lim _{p \rightarrow \infty}\left(1+\sum_{j=1}^{\lfloor p / 2\rfloor} \alpha_{2 j}(-1)^{j} \lambda^{j} h^{2 j}\right)=1+\sum_{j=1}^{\infty} \frac{1}{(2 j) !} \frac{1}{2^{2 j}}(-1)^{j} \lambda^{j} h^{2 j} \\
& =\sum_{j=0}^{\infty}(-1)^{j}\left(\frac{\sqrt{\lambda} h}{2}\right)^{2 j} \frac{1}{(2 j) !}=\cos (\sqrt{\lambda}) \frac{h}{2} .
\end{aligned}
$$

By using the same idea, we obtain

$$
\lim _{p \rightarrow \infty} m_{12}=\lim _{p \rightarrow \infty}\left(\sum_{j=0}^{\lfloor(p-1) / 2\rfloor}(-1)^{j} \alpha_{2 j+1} \lambda^{j} h^{2 j+1}\right)=\frac{1}{\sqrt{\lambda}} \sin (\sqrt{\lambda}) \frac{h}{2}
$$

It follows from (2.28) and (2.29) that

$$
\lim _{p \rightarrow \infty} \operatorname{det}(M)=m_{22}^{2}+\lambda m_{12}^{2}=\cos ^{2}(\sqrt{\lambda}) \frac{h}{2}+\lambda\left(\frac{1}{\sqrt{\lambda}} \sin (\sqrt{\lambda}) \frac{h}{2}\right)^{2}=1
$$

Hence, for $r(x)=0$, the approximate eigenfunction of (1.1) to the corresponding eigenvalue $\lambda$ converges to exact eigenfunction:

$$
\lim _{p \rightarrow \infty} y_{1}=2 \frac{z_{0}}{\operatorname{det}(M)} \frac{1}{\sqrt{\lambda}}\left(\cos \left(\sqrt{\lambda} \frac{h}{2}\right)\right)\left(\sin \left(\sqrt{\lambda} \frac{h}{2}\right)\right)=\frac{z_{0}}{\sqrt{\lambda}} \sin \left(\sqrt{\lambda}\left(\bar{x}-x_{0}\right)\right) .
$$

Since we have $z(x)=y^{\prime}(x)$, the derivative of approximate eigenfunction of (1.1) to the corresponding eigenvalue $\lambda$ converges to derivative of the exact solution:

$$
\lim _{p \rightarrow \infty} z_{1}=\frac{z_{0}}{\operatorname{det}(M)}\left((-\lambda) \frac{1}{\lambda} \sin ^{2}\left(\sqrt{\lambda} \frac{h}{2}\right)+\cos ^{2}\left(\sqrt{\lambda} \frac{h}{2}\right)\right)=z_{0} \cos \left(\sqrt{\lambda}\left(\bar{x}-x_{0}\right)\right),
$$

where $\lambda=k^{2} \pi^{2}, k=1,2, \ldots$

This demonstration shows that approximate eigenfunction and eigenvalue converges to exact one as $p \rightarrow \infty$ for fixed step-size " $h . "$ 


\subsection{Taylor's Decomposition Method to the Euler Buckling Problem}

For convenience, we introduce the following notations as in (2.1) and Adiyaman and Somali [7]:

$$
\begin{aligned}
& Y(x)=\left[\begin{array}{l}
y(x) \\
z(x)
\end{array}\right], \quad F(Y(x))=\left[\begin{array}{l}
f_{1}^{(0)}(y, z) \\
f_{2}^{(0)}(y, z)
\end{array}\right], \\
& C_{0}=\left[\begin{array}{ll}
0 & 1 \\
0 & 0
\end{array}\right], \quad C_{1}=\left[\begin{array}{ll}
0 & 0 \\
0 & 1
\end{array}\right], \quad f_{1}^{(0)}(y, z)=z, \quad f_{2}^{(0)}(y, z)=-\lambda \sin y \text {. }
\end{aligned}
$$

Thus, the Euler Buckling Problem (1.2) can be written in the form:

$$
\begin{gathered}
Y^{\prime}(x)=F(Y(x)), \quad 0<x<1, \\
C_{0} Y(0)+C_{1} Y(1)=0,
\end{gathered}
$$

Defining the following recurrence relations for $j=1, \ldots, 2 p$ :

$$
f_{i}^{(j)}(y, z)=z \frac{\partial f_{i}^{(j-1)}(y, z)}{\partial y}-\lambda \sin y \frac{\partial f_{i}^{(j-1)}(y, z)}{\partial z}, i=1,2
$$

we obtain

$$
Y^{(j)}(x)=\left[\begin{array}{l}
f_{1}^{(j-1)}(y, z) \\
f_{2}^{(j-1)}(y, z)
\end{array}\right]=\left[\begin{array}{l}
f_{2}^{(j-2)}(y, z) \\
f_{2}^{(j-1)}(y, z)
\end{array}\right] \text { for } j=2, \ldots, 2 p+1
$$

We first give the following lemma which defines $f_{2}^{(j-1)}(y, z)$ explicitly.

Lemma 2.1. For $j=0, \ldots, 2 p$, let $f_{2}^{(j)}(y, z)$ satisfy the recurrence relation (2.35) with $f_{2}^{(0)}(y, z)=$ $-\lambda \sin y$. Then

$$
f_{2}^{(2 m)}(y, z)=\sum_{i=0}^{m} \lambda^{i+1} z^{2 m-2 i} \sum_{k=0}^{\lfloor i / 2\rfloor}(-1)^{m+1-k} a_{2 m+1, i, k}(\cos y)^{i-2 k}(\sin y)^{2 k+1}
$$

where

$$
a_{2 m+1, i, k}= \begin{cases}1, & i=0, k=0, \\ (2 k+2) a_{2 m, i, k+1}+(i+1-2 k) a_{2 m, i, k} & \\ +(2 m+1-2 i) a_{2 m, i-1, k}, & 1 \leq i \leq m, 0 \leq k \leq \frac{i}{2} \\ 0, & \text { else, }\end{cases}
$$


for $m=0, \ldots, p$, and

$$
f_{2}^{(2 m+1)}(y, z)=\sum_{i=0}^{m} \lambda^{i+1} z^{2 m+1-2 i} \sum_{k=0}^{\lfloor(i+1) / 2\rfloor}(-1)^{m+1-k} a_{2 m+2, i, k}(\cos y)^{i+1-2 k}(\sin y)^{2 k}
$$

where

$$
a_{2 m+2, i, k}= \begin{cases}1, & i=0, k=0, \\ (2 k+1) a_{2 m+1, i, k}+(i-2 k) a_{2 m+1, i, k-1} & \\ +(2 m+2-2 i) a_{2 m+1, i-1, k-1,} & 1 \leq i \leq m, 0 \leq k \leq \frac{i}{2} \\ 0, & \text { else, }\end{cases}
$$

for $m=0, \ldots, p-1$.

Proof. The proof follows induction argument based on (2.35).

Theorem 2.2. If $f_{1}^{(j)}(y, z)$ and $f_{2}^{(j)}(y, z)$, are sufficiently smooth and satisfy (2.36), (2.37), and (2.39) then the following relations hold: (a) it holds that

$$
\begin{gathered}
y_{1}-y_{0}+\sum_{j=1}^{p} \beta_{j}\left[(-1)^{j} f_{1}^{(j-1)}\left(y_{1}, 0\right)-f_{1}^{(j-1)}\left(y_{0}, 0\right)\right]=-2\left(y_{0}+\sum_{j=1}^{p} \beta_{j} f_{1}^{(j-1)}\left(y_{0}, 0\right)\right), \\
\sum_{j=1}^{p} \beta_{j}\left[(-1)^{j} f_{2}^{(j-1)}\left(y_{1}, 0\right)-f_{2}^{(j-1)}\left(y_{0}, 0\right)\right]=0,
\end{gathered}
$$

for $y_{1}=-y_{0},(b)$ it holds that

$$
\begin{gathered}
\sum_{j=1}^{p} \beta_{j}\left[(-1)^{j} f_{2}^{(j-1)}\left(y_{1}, 0\right)-f_{2}^{(j-1)}\left(y_{0}, 0\right)\right]=-2 \sum_{j=1}^{p} \beta_{j} f_{2}^{(j-1)}\left(y_{0}, 0\right), \\
y_{1}-y_{0}+\sum_{j=1}^{p} \beta_{j}\left[(-1)^{j} f_{1}^{(j-1)}\left(y_{1}, 0\right)-f_{1}^{(j-1)}\left(y_{0}, 0\right)\right]=0,
\end{gathered}
$$

for $y_{1}=y_{0}$.

Proof. Let $j=2 m+1$ for $m=0, \ldots, p$, then $f_{1}^{(2 m+1)}(y, z)$ becomes

$$
f_{1}^{(2 m)}(y, z)=f_{2}^{(2 m-1)}(y, z)=\sum_{i=0}^{(m-1)} \lambda^{i+1} z^{2 m-2 i-1} \sum_{k=0}^{\lfloor(i+1) / 2\rfloor}(-1)^{m-k} a_{2 m, i, k}(\cos y)^{i+1-2 k}(\sin y)^{2 k}
$$


by Lemma 2.1. Since all terms of previous sum contain $z, f_{1}^{2 m}(y, 0)=f_{2}^{2 m-1}(y, 0)=0$ for $m=1, \ldots, p$, hence, we get the following equations:

$$
\begin{gathered}
y_{1}-y_{0}+\sum_{j=1}^{p} \beta_{j}\left[(-1)^{j} f_{1}^{(j-1)}\left(y_{1}, 0\right)-f_{1}^{(j-1)}\left(y_{0}, 0\right)\right] \\
=y_{1}-y_{0}+\sum_{j=1}^{p} \beta_{j}\left[f_{1}^{(j-1)}\left(y_{1}, 0\right)-f_{1}^{(j-1)}\left(y_{0}, 0\right)\right], \\
\sum_{j=1}^{p} \beta_{j}\left[(-1)^{j} f_{2}^{(j-1)}\left(y_{1}, 0\right)-f_{2}^{(j-1)}\left(y_{0}, 0\right)\right]=\sum_{j=1}^{p} \beta_{j}\left[-f_{2}^{(j-1)}\left(y_{1}, 0\right)-f_{2}^{(j-1)}\left(y_{0}, 0\right)\right] .
\end{gathered}
$$

Letting $j=2 m+2$ for $m=0, \ldots, p-1$ and using (2.37), we get

$$
f_{1}^{(2 m+2-1)}(y, z)=f_{2}^{(2 m)}(y, z)=\sum_{i=0}^{m} \lambda^{i+1} z^{2 m-2 i} \sum_{k=0}^{\lfloor i / 2\rfloor}(-1)^{m+1-k} a_{2 m+1, i, k}(\cos y)^{i-2 k}(\sin y)^{2 k+1}
$$

Substituting the value $z=0$ into (2.45), we obtain

$$
\begin{aligned}
f_{1}^{(2 m+1)}\left(-y_{0}, 0\right) & =f_{2}^{2 m}\left(-y_{0}, 0\right)=\lambda^{m+1} \sum_{k=0}^{\lfloor m / 2\rfloor}(-1)^{m+1-k} a_{2 m+1, m, k}\left(\cos y_{0}\right)^{i-2 k}\left[-\left(\sin y_{0}\right)^{2 k+1}\right] \\
& =-f_{2}^{(2 m)}\left(y_{0}, 0\right),
\end{aligned}
$$

which gives the following relations:

$$
\begin{gathered}
f_{1}^{(2 m+1)}\left(-y_{0}, 0\right)=-f_{1}^{(2 m+1)}\left(y_{0}, 0\right), \\
f_{2}^{(2 m)}\left(-y_{0}, 0\right)=-f_{2}^{(2 m)}\left(y_{0}, 0\right) .
\end{gathered}
$$


Table 1: Corresponding to the initial values $y_{1,0}, y_{2,0}, y_{3,0}$, and $y_{4,0}$ for various $\lambda$ obtained from (2.54) and (2.55).

\begin{tabular}{lcccc}
\hline$\lambda$ & $y_{1,0}$ & $y_{2,0}$ & $y_{3,0}$ & $y_{4,0}$ \\
\hline $15>\pi^{2}$ & 1.7471 & - & - & - \\
$45>4 \pi^{2}$ & 2.8578 & 1.0092 & - & - \\
$90>9 \pi^{2}$ & 3.0718 & 2.3413 & 0.3236 & - \\
$160>16 \pi^{2}$ & 3.1272 & 2.7999 & 2.0239 & 0.3771 \\
\hline
\end{tabular}

Using (2.47) for $y_{1}=-y_{0}$, we obtain the following relations:

$$
\begin{aligned}
& y_{1}-y_{0}+\sum_{j=1}^{p} \beta_{j}\left[(-1)^{j} f_{1}^{(j-1)}\left(y_{1}, 0\right)-f_{1}^{(j-1)}\left(y_{0}, 0\right)\right] \\
&=-y_{0}-y_{0}+\sum_{j=1}^{p} \beta_{j}\left[f_{1}^{(j-1)}\left(-y_{0}, 0\right)-f_{1}^{(j-1)}\left(y_{0}, 0\right)\right] \\
&=-2\left(y_{0}+\sum_{j=1}^{p} \beta_{j} f_{1}^{(j-1)}\left(y_{0}, 0\right)\right) \\
& \sum_{j=1}^{p} \beta_{j}\left[-f_{2}^{(j-1)}\left(y_{1}, 0\right)-f_{2}^{(j-1)}\left(y_{0}, 0\right)\right]=0 .
\end{aligned}
$$

Similarly for $y_{1}=y_{0}$ using (2.47), we observe that

$$
\begin{gathered}
y_{1}-y_{0}+\sum_{j=1}^{p} \beta_{j}\left[(-1)^{j} f_{1}^{(j-1)}\left(y_{1}, 0\right)-f_{1}^{(j-1)}\left(y_{0}, 0\right)\right]=0, \\
\sum_{j=1}^{p} \beta_{j}\left[(-1)^{j} f_{2}^{(j-1)}\left(y_{1}, 0\right) f_{2}^{(j-1)}\left(y_{0}, 0\right)\right]=-2 \sum_{j=1}^{p} \beta_{j} f_{2}^{(j-1)}\left(y_{0}, 0\right) .
\end{gathered}
$$

So, our assertions (a) and (b) are proved.

Again, we consider the application of Taylor's decomposition method to (2.34) on two points $x_{k}$ and $x_{k-1}$ :

$$
Y_{k}-Y_{k-1}+\sum_{j=1}^{p} \alpha_{j} Y_{k}^{(j)} h^{j}-\sum_{j=1}^{q} \beta_{j} Y_{k-1}^{(j)} h^{j}=0
$$

where $Y_{k}^{(j)}$ is the approximate value of $Y_{k}^{(j)}\left(x_{k}\right)$. For the computation of the eigenvalues of (1.2), putting $h=1$ and $p=q$, the approximation (2.50) gives

$$
Y_{1}-Y_{0}+\sum_{j=1}^{p}(-1)^{j} \beta_{j} Y_{1}^{(j)}-\sum_{j=1}^{p} \beta_{j} Y_{0}^{(j)}=0,
$$


Table 2: Comparison of the first eigenvalue and solutions of Example 3.1 using Taylor's decomposition method, exact values, and Table 4 from [9], when $n=0$.

\begin{tabular}{|c|c|c|c|c|c|c|c|}
\hline$x$ & $\begin{array}{c}\text { HWSM } \\
(h=1 / 16)\end{array}$ & $\begin{array}{c}\text { FDM } \\
(h=1 / 16)\end{array}$ & $\begin{array}{c}\text { TDM } \\
(p=3) \\
(h=1 / 16)\end{array}$ & Exact & $\begin{array}{c}\text { Errors of } \\
\text { TDM } \\
(p=3) \\
(h=1 / 16)\end{array}$ & $\begin{array}{c}\text { Errors of } \\
\text { TDM } \\
(p=4) \\
(h=1 / 8)\end{array}$ & $\begin{array}{c}\text { Errors of } \\
\text { TDM } \\
(p=5) \\
(h=1 / 4)\end{array}$ \\
\hline 0 & 0 & 0 & 0 & 0 & 0 & 0 & 0 \\
\hline 0.0625 & 0.27521 & 0.278599 & 0.275999 & 0.275899 & $1.54 E-10$ & - & - \\
\hline 0.125 & 0.54181 & 0.541196 & 0.541196 & 0.541196 & $2.91 E-10$ & $1.13 E-11$ & - \\
\hline 0.1875 & 0.78549 & 0.785695 & 0.785695 & 0.785695 & $3.93 E-10$ & - & - \\
\hline 0.25 & 1.00482 & 1 & 1 & 1 & $4.45 E-10$ & $1.74 E-11$ & $6.87 E-12$ \\
\hline 0.3125 & 1.17851 & 1.17588 & 1.17588 & 1.17588 & $4.37 E-10$ & - & - \\
\hline 0.375 & 1.31285 & 1.30656 & 1.30656 & 1.30656 & $3.61 E-10$ & $1.41 E-11$ & - \\
\hline 0.4375 & 1.38376 & 1.38704 & 1.38704 & 1.38704 & $2.15 E-10$ & - & - \\
\hline 0.5 & 1.41103 & 1.41421 & 1.41421 & 1.41421 & $2.22 E-16$ & $2.22 E-16$ & 0 \\
\hline 0.5625 & 1.38376 & 1.38704 & 1.38704 & 1.38704 & $2.76 E-10$ & - & - \\
\hline 0.625 & 1.31285 & 1.30656 & 1.30656 & 1.30656 & $6.03 E-10$ & $2.35 E-11$ & - \\
\hline 0.6875 & 1.17851 & 1.17588 & 1.17588 & 1.17588 & $9.63 E-10$ & - & - \\
\hline 0.75 & 1.00482 & 1 & 1 & 1 & $1.33 E-9$ & $5.22 E-11$ & 2.06E-11 \\
\hline 0.8125 & 0.78549 & 0.785695 & 0.785695 & 0.785695 & $1.70 E-9$ & - & - \\
\hline 0.875 & 0.54181 & 0.541196 & 0.541196 & 0.541196 & $2.03 E-9$ & $7.96 E-11$ & - \\
\hline 0.9375 & 0.27521 & 0.275899 & 0.275999 & 0.275899 & $2.31 E-9$ & - & - \\
\hline 1 & 0 & 0 & 0 & 0 & 0 & 0 & 0 \\
\hline
\end{tabular}

Table 3: Comparison of the first eigenvalue and solutions of Example 3.1 using TDM and Table 4 from [9], when $p=16, n=2$, and $h=0.0625$.

\begin{tabular}{lccc}
\hline$x$ & HWSM & FDM & TDM \\
\hline 0 & 0 & 0 & 0 \\
0.0625 & 0.27521 & 0.27756 & 0.277563 \\
0.125 & 0.54181 & 0.54434 & 0.544337 \\
0.1875 & 0.78949 & 0.78996 & 0.789953 \\
0.25 & 1.00485 & 1.00488 & 1.00487 \\
0.3125 & 1.18153 & 1.18075 & 1.18074 \\
0.375 & 1.31286 & 1.31082 & 1.31076 \\
0.4375 & 1.39372 & 1.38996 & 1.38994 \\
0.5 & 1.42102 & 1.41527 & 1.41529 \\
0.5625 & 1.39371 & 1.38591 & 1.38598 \\
0.625 & 1.31285 & 1.30323 & 1.30334 \\
0.6875 & 1.18154 & 1.18066 & 1.17081 \\
0.75 & 1.0048 & 0.99361 & 0.993792 \\
0.8125 & 0.77949 & 0.77917 & 0.779357 \\
0.875 & 0.53481 & 0.53577 & 0.535934 \\
0.9375 & 0.27726 & 0.27277 & 0.272878 \\
1 & 0 & 0 & 0 \\
\hline$\lambda_{1}=10.3452$ (HWSM) $9.95067($ FDM) & $9.98317(\mathrm{TDM})$ & &
\end{tabular}

$\lambda_{1}=10.3452$ (HWSM), 9.95067 (FDM), 9.98317 (TDM). 
Table 4: The errors between exact and approximate fundamental eigenvalue for various $p$ and for $h=1$ and $n=0$ in Example 3.1.

\begin{tabular}{lcccccccc}
\hline$p$ & 2 & 3 & 4 & 6 & 7 & 9 & 10 & 11 \\
\hline Errors & $2.13 E 0$ & $1.30 E-1$ & $5.49 E-3$ & $2.60 E-6$ & $3.38 E-8$ & $2.60 E-12$ & $1.59 E-14$ & $1.77 E-15$ \\
\hline
\end{tabular}

Table 5: Observed orders of Example 3.1 for $n=2$ at $x=1 / 2$ using Taylor's decomposition method.

\begin{tabular}{lccc}
\hline & $p=2$ & $p=3$ & $p=4$ \\
\hline $\operatorname{ord}(1 / 8)$ & 4.23247 & 7.90607 & 10.6574 \\
$\operatorname{ord}(1 / 16)$ & 3.86153 & 5.22141 & 8.13719 \\
$\operatorname{ord}(1 / 32)$ & 3.98674 & 5.93074 & 7.63932 \\
\hline
\end{tabular}

where $\alpha_{j}=(-1)^{j} \beta_{j}$. Writing (2.51) with respect to the components and imposing the boundary conditions $z_{0}=z(0)=y^{\prime}(0)=0$ and $z_{1}=z(1)=y^{\prime}(1)=0$, we have the following equations

$$
\begin{gathered}
y_{1}-y_{0}+\sum_{j=1}^{p} \beta_{j}\left[(-1)^{j} f_{1}^{(j-1)}\left(y_{1}, 0\right)-f_{1}^{(j-1)}\left(y_{0}, 0\right)\right]=0, \\
\sum_{j=1}^{p} \beta_{j}\left[(-1)^{j} f_{2}^{(j-1)}\left(y_{1}, 0\right)-f_{2}^{(j-1)}\left(y_{0}, 0\right)\right]=0 .
\end{gathered}
$$

Using Theorem 2.2(a) for $y_{1}=-y_{0},(2.52)$ becomes

$$
G_{1}\left(y_{0}, \lambda\right)=-2\left(y_{0}+\sum_{j=1}^{p} \beta_{j} f_{1}^{(j-1)}\left(y_{0}, 0\right)\right)=0
$$

and (2.53) is satisfied. For $y_{1}=y_{0},(2.52)$ is satisfied by Theorem 2.2(b) and (2.53) becomes

$$
G_{2}\left(y_{0}, \lambda\right)=-2 \sum_{j=1}^{p} \beta_{j} f_{2}^{(j-1)}\left(y_{0}, 0\right)=0
$$

From Table 1, we observe that there is only trivial initial condition for $0 \leq \lambda \leq \pi^{2}$, there is one nontrivial initial condition from (2.54) for $\pi^{2}<\lambda \leq 4 \pi^{2}$, there are $n$ nontrivial initial conditions for $n^{2} \pi^{2}<\lambda \leq(n+1)^{2} \pi^{2}$. These results show that the numerical results obtained using Taylor's decomposition method agree with the theoretical results of Euler buckling problem given in [2].

Now, we find an approximate solution to the problem

$$
\begin{gathered}
Y^{\prime}(x)=F(Y(x)) \\
Y(0)=Y_{0}
\end{gathered}
$$

Which corresponds to Euler buckling problem (1.2) for an eigenvalue $\lambda$ and the initial value $y_{0}$. Using Taylor's Decomposition on two points $x_{k-1}, x_{k}$ for $p=q$ then $y_{0} \simeq y(0)$, 
Table 6: Comparison of higher eigenvalues for Mathieu's equation obtained from FDM, HWSM, and TDM corresponding to $\theta=5$.

\begin{tabular}{ccccc}
\hline$n$ & $n^{2}$ & $\lambda_{n}(\mathrm{FDM})$ & $\lambda_{n}(\mathrm{HWSM})$ & $\lambda_{n}(\mathrm{TDM})$ \\
\hline 1 & 1 & -5.7311 & -5.4665 & -5.79008 \\
2 & 4 & 2.0992 & 2.6161 & 2.09946 \\
3 & 9 & 9.2365 & 9.4227 & 9.23633 \\
4 & 16 & 16.648 & 16.3707 & 16.6482 \\
5 & 25 & 25.511 & 24.1471 & 25.5108 \\
6 & 36 & 36.359 & 36.6577 & 36.3589 \\
\hline
\end{tabular}

$\lambda_{1}=-5.46653$ (HWSM), -5.73115 (FDM), -5.79008 (TDM).

Table 7: Comparison of the exact eigenvalues with approximate eigenvalues obtained from Numerov's method $\left(\Lambda_{k}\right)$ with correction and TDM $\left(\mu_{k}\right)$ for Example 3.3 corresponding to $\beta=10$.

\begin{tabular}{cccccc}
\hline$k$ & $\lambda_{k}$ & $\lambda_{k}-\Lambda_{k}^{(40)}$ & $\lambda_{k}-\Lambda_{k}^{(80)}$ & $\lambda_{k}-\mu_{k}^{70}$ & $\lambda_{k}-\mu_{k}^{80}$ \\
\hline 1 & 0.0000000 & $2.32 E-3$ & $1.43 E-4$ & $3.63 E-3$ & $7.60 E-5$ \\
2 & 37.7596285 & $7.52 E-3$ & $4.42 E-4$ & $9.15 E-4$ & $3.81 E-6$ \\
3 & 37.8059002 & $1.61 E-3$ & $9.93 E-5$ & $5.66 E-7$ & $1.12 E-7$ \\
4 & 37.8525995 & $6.88 E-3$ & $4.43 E-4$ & $8.97 E-4$ & $3.85 E-5$ \\
5 & 70.5475097 & $3.22 E-2$ & $1.97 E-3$ & $1.57 E-2$ & $3.74 E-4$ \\
6 & 92.6538177 & $2.25 E-2$ & $1.37 E-3$ & $2.25 E-3$ & $2.20 E-5$ \\
7 & 96.2058159 & $1.20 E-2$ & $7.28 E-4$ & $2.18 E-3$ & $1.78 E-4$ \\
8 & 102.254347 & $3.45 E-2$ & $2.09 E-3$ & $4.38 E-3$ & $2.44 E-4$ \\
\hline
\end{tabular}

$h=\pi$ for Taylor's decomposition method.

$z_{0}=z(0)$. Solving the obtained nonlinear system by Newton's method, we obtain the approximate value $y_{k}$ of the eigenfunction $y(x)$ at $x=x_{k}$ with $O\left(h^{2 p}\right)$.

It is clear that $f_{2}^{(0)}(y, z)=\sin y$ is Lipschitz in $y$ in 2-dimensional box $D$. Using the results (Adiyaman and Somali [7, Lemma 2 and Theorem 3]), the global error for (2.50) is bounded by

$$
\left\|Y\left(x_{k}\right)-Y_{k}\right\| \leq C_{0}\left\|Y(0)-Y_{0}\right\|+C_{1} \frac{\xi h^{2 p} M^{p+1}}{(2 p) !}
$$

where $C_{0}=e^{\bar{x}((2 L B(h)) /(1-L B(h)))}, C_{1}=$ const $\left(C_{0} / L\right)\left(1 /\left(1+\left(\beta_{2} / \beta_{1}\right) h+\cdots+\beta_{p} / \beta_{1} h^{p-1}\right)\right), M=$ $\max _{(y, z) \in D}\left\{\left|f_{1}^{(0)}(y, z)\right|,\left|f_{2}^{(0)}(y, z)\right|\right\}, D$ is 2-dimensional box in $R^{2}, \xi=\max \left\{\sum_{k=0}^{\lfloor(i+1) / 2\rfloor} a_{j, i, k}\right\}$, $j=1, \ldots, 2 p, i=0, \ldots, p$, const is a constant independent of $h, p,\|\cdot\|$ denotes $\|\cdot\|_{\infty}$, $L=\max _{1 \leq j \leq p}\left\{l_{1, j}, l_{2, j}\right\}$ with $l_{1, j}=\max _{1 \leq j \leq p}\left\{d_{1, j}, s_{1, j}\right\}, l_{2, j}=\max _{1 \leq j \leq p}\left\{d_{2, j}, s_{2, j}\right\}, d_{k, j}=$ $\max _{(y, z) \in D}\left|\left(\partial f_{k}^{(j)}(y, z) / \partial y\right)\right|, s_{k, j}=\max _{(y, z) \in D}\left|\left(\partial f_{k}^{(j)}(y, z) / \partial z\right)\right|, k=1,2$, and $B(h)=$ $L \sum_{j=1}^{p} \beta_{j} h^{j-1}$ for some $\bar{x}>0$. 
Table 8: Comparison of the exact eigenvalues with approximate eigenvalues obtained from Numerov's method $\left(\Lambda_{k}\right)$ with correction and TDM $\left(\mu_{k}\right)$ for Example 3.3 corresponding to $\beta=20$.

\begin{tabular}{cccccc}
\hline$k$ & $\lambda_{k}$ & $\lambda_{k}-\Lambda_{k}^{(40)}$ & $\lambda_{k}-\Lambda_{k}^{(80)}$ & $\lambda_{k}-\mu_{k}^{100}$ & $\lambda_{k}-\mu_{k}^{110}$ \\
\hline 1 & 0.0000000 & $1.93 E-2$ & $1.17 E-3$ & $1.04 E-1$ & $7.64 E-3$ \\
2 & 77.9161943 & $1.22 E-1$ & $7.38 E-3$ & $4.37 E-2$ & $6.98 E-2$ \\
3 & 77.9161957 & $1.63 E-2$ & $9.95 E-4$ & $9.89 E-4$ & $1.67 E-4$ \\
4 & 77.9161972 & $1.62 E-2$ & $9.89 E-4$ & $1.13 E-1$ & $7.48 E-2$ \\
5 & 151.463224 & $3.90 E-1$ & $2.33 E-2$ & $7.04 E-1$ & $1.56 E-1$ \\
\hline
\end{tabular}

$h=\pi$ for Taylor's decomposition method.

\section{Numerical Results}

\subsection{Numerical Results for Regular Sturm-Liouville Eigenvalue Problems}

We consider three regular Sturm-Liouville eigenvalue problems, one of them has polynomial coefficients and the others have periodic coefficients taken from Bujurke et al. [9] and Andrew [10].

Example 3.1. Consider the Titchmarch equation:

$$
\begin{gathered}
y^{\prime \prime}+\left(\lambda-x^{2 n}\right) y(x)=0, \\
y(0)=y(1)=0,
\end{gathered}
$$

where $n$ is a nonnegative integer. We obtain the numerical solutions taking $n=0,2$. The accuracy of the method is tested by comparing with the exact solution which exists when $n=0$ and finite-difference method (FDM) solution and Haar wavelet series method (HWSM) solution when $n=2$.

Tables 2 and 3 give computed eigenvalues and solution $y(x)$ of Titchmarch problem using Taylor's decomposition method (TDM) with different values of $p$, HWSM and FDM for $n=0,2$, the integer parameter in Titchmarch problem. In Table 2, it is easily seen that the error between approximate eigenfunction and exact eigenfunction decreases as $p$ increases or the step-size decreases or both happen. So, we can find good approximation to eigenfunctions for relatively large step-sizes by increasing $p$. In Table $3 m$ is the number of intervals. Table 4 gives the errors between exact and approximate eigenvalues for fixed step-size $h=1$ for $n=0$. Notice that, as $p$ increases, the accuracy of approximation almost doubles in digits which demonstrates a fast convergence.

Example 3.2. Consider the Mathieu's equation:

$$
\begin{gathered}
y^{\prime \prime}+(\lambda-2 \theta \cos (2 x)) y=0, \\
y(0)=y(\pi)=0 .
\end{gathered}
$$

We will solve these two problems approximately using Taylor's decomposition method (TDM), and we will compare our results with the results in Bujurke et al. [9]. Bujurke et al. [9] solved Examples 3.1 and 3.2 approximately using Haar wavelets. They transform 


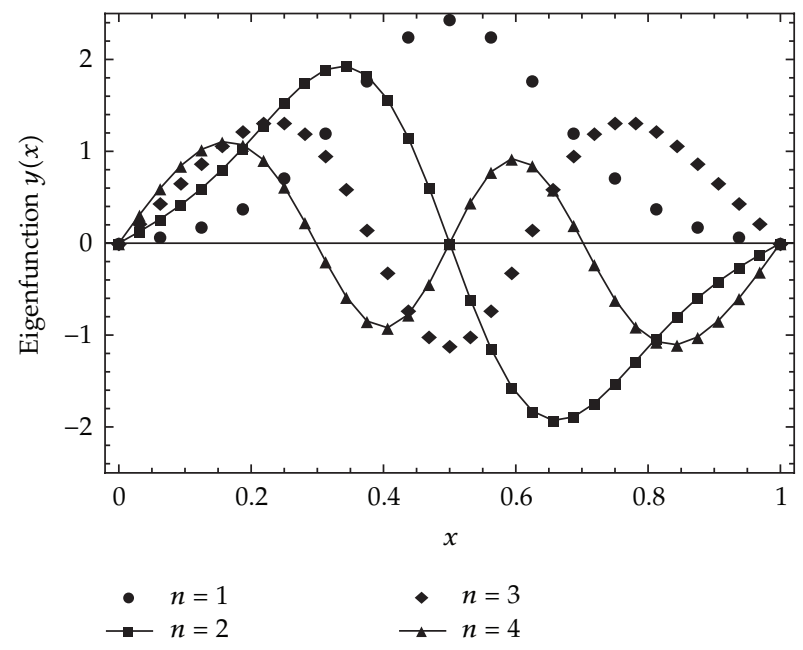

Figure 1: Higher eigenfunctions of Mathieu's equation for a fixed parameter $\theta=5$.

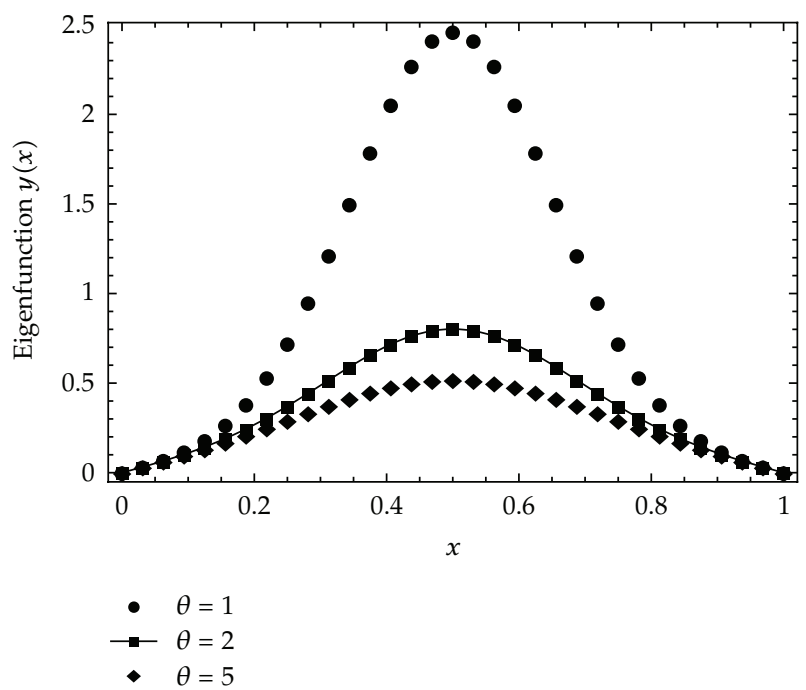

Figure 2: Solutions of Mathieu's equation for different parameters of $\theta$.

the interval $[0, \pi]$ to $[0,1]$ because of the properties of Haar wavelets. So to compare the results we normalize the interval $[0, \pi]$ by using $x=\pi t$, Mathieu's equation in Example 3.2 transformed into

$$
\begin{gathered}
y^{\prime \prime}+\left(\pi^{2} \lambda-2 \pi^{2} \theta \cos (2 \pi t)\right) y=0 \\
y(0)=y(1)=0 .
\end{gathered}
$$

The eigenvalues for a fixed value for $\theta=5$ are obtained in Table 6 which gives the asymptotic behavior of higher eigenvalues of Mathieu's equation, and these eigenvalues are $\lambda_{n}=n^{2}+O(1)$. This result agrees with the classical theorem on asymptoticity of the 


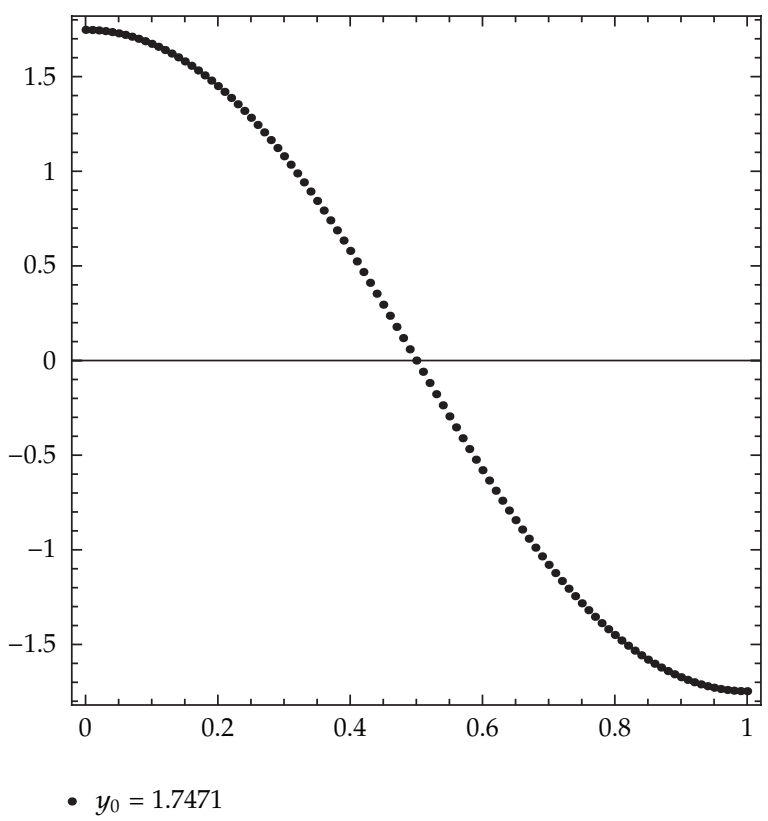

Figure 3: Solution of (1.2) corresponding to the initial values $y_{0}$ for $\pi^{2} \leq \lambda=15<4 \pi^{2}$.

eigenvalues $\lim _{n \rightarrow \infty} \lambda_{n}^{1 / 2} / n=1$ from van Brunt [11]. Figure 1 demonstrates that the $n$th eigenfunction has $n-1$ zeros in $(0,1)$ which is consistent with the relevant graph in Bujurke et al. [9]. The selected values of parameter $\theta$ shifts the symmetry of the solutions and this property is given in Figure 2.

Example 3.3. Consider the equation

$$
\begin{gathered}
-y^{\prime \prime}+\left(2 \beta \cos 2 x+\beta^{2} \sin ^{2} 2 x\right) y=\lambda y, \\
y^{\prime}(0)=y^{\prime}(\pi)=0 .
\end{gathered}
$$

We give the comparison of approximate eigenvalues obtained using Taylor's Decomposition method with the approximate eigenvalues obtained using Numerov's method Andrew [10] for $\beta=10$ and $\beta=20$ in Tables 7 and 8 . The values shown as the "exact" $\lambda_{k}$ and the corrected approximate eigenvalues $\Lambda_{k}$ obtained using Numerov's method for step-sizes $h=40$ and $h=80$ in Tables 7 and 8 are taken from Andrew [10]. The values shown as $\mu_{k}$ are evaluated using Taylor's Decomposition method for $p=70$ and 80 in Table 7 and for $p=100$ and 110 in Table 8. From the tables, it can be seen that Taylor's Decomposition method approximates small eigenvalues with high-order accuracy without using any correction.

In comparison to Example 3.1, the estimation of eigenvalues for Examples 3.2 and 3.3 is more complicated. But Example 3.1 is important to show the high accuracy of the method while calculating the eigenfunctions for relatively large step-sizes. Other two examples show the accuracy of the method while calculating the eigenvalues for large step-sizes which equal to whole interval. 


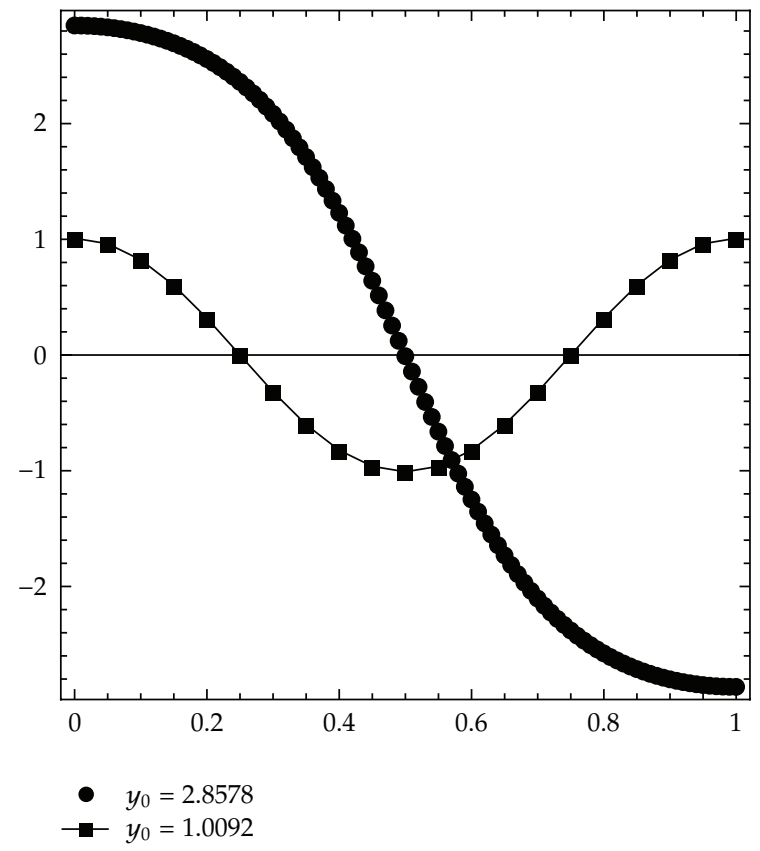

Figure 4: Solution of (1.2) corresponding to the initial values $y_{0}$ for $4 \pi^{2} \leq \lambda=45<9 \pi^{2}$.

In Table 5, the observed orders ord $(h)$ are computed using the following formula

$$
\operatorname{ord}(h)=\frac{\log \left(\left(y_{4 h}-y_{2 h}\right) /\left(y_{2 h}-y_{h}\right)\right)}{\log 2}
$$

where $y_{4 h}, y_{2 h}$, and $y_{h}$ are the approximated value of eigenfunctions at $x_{k}$ to the corresponding eigenvalue $\lambda$ when the problems are solved with step sizes $4 h, 2 h$, and $h$ respectively. The observed orders given in Table 5 well confirm the theoretical results. That is, the order of TDM is order of $2 p$.

The numerical calculations and all figures in this work are performed using Mathematica.

\subsection{Numerical Results for Euler Buckling Problem}

The approximate solutions of Euler Buckling problem for $\lambda=15, \lambda=45, \lambda=90$, and $\lambda=160$ generated using Taylor's Decomposition method for step size $h=1 / 20$ are illustrated in Figures 3, 4, 5, and 6, respectively.

\section{Conclusion}

In this paper, we have described Taylor's Decomposition method for regular SturmLiouville eigenvalue problems with Dirichlet and Neumann boundary conditions to obtain approximate eigenvalues and eigenfunctions and for Euler Buckling Problem to obtain 


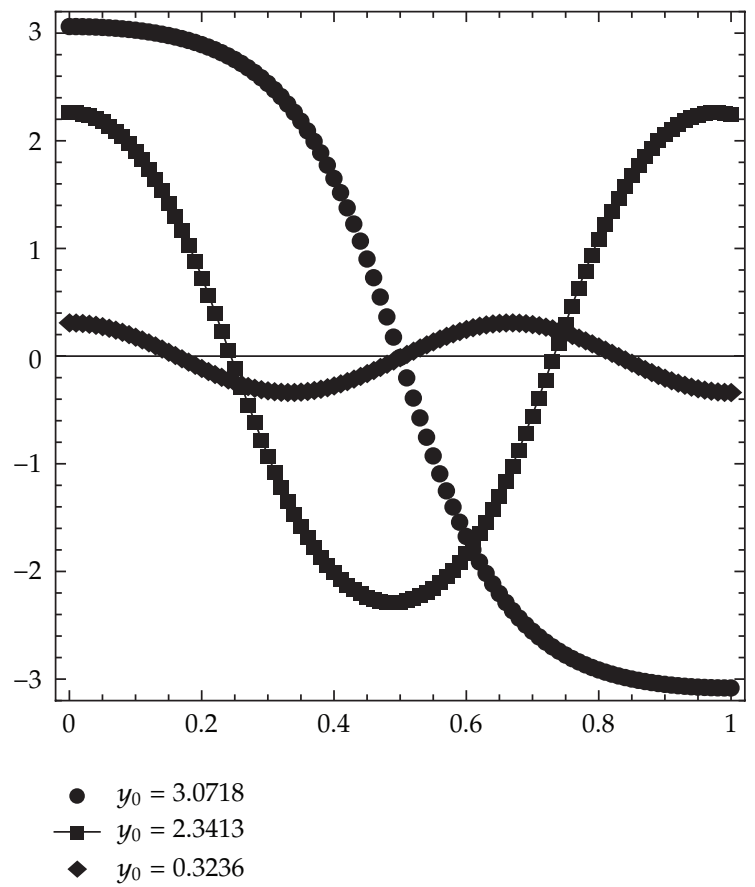

Figure 5: Solution of (1.2) corresponding to the initial values $y_{0}$ for $9 \pi^{2} \leq \lambda=90<16 \pi^{2}$.

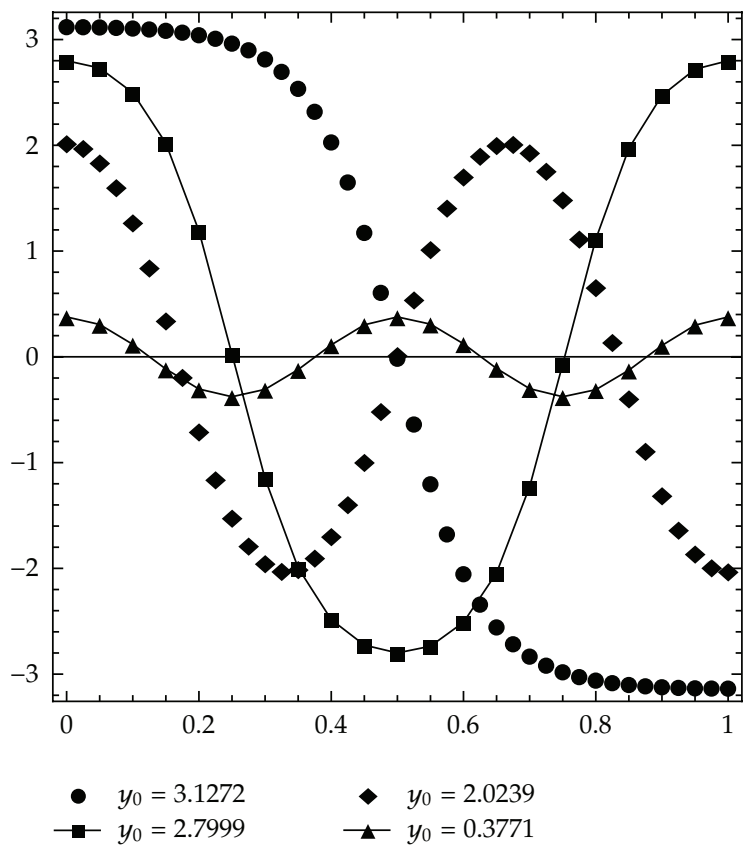

Figure 6: Solution of (1.2) corresponding to the initial values $y_{0}$ for $16 \pi^{2} \leq \lambda=160<25 \pi^{2}$. 
approximate initial values and eigenfunctions. The obtained results for Euler Buckling problem give the behavior of eigenvalues and corresponding eigenfunctions with highorder accuracy without using small stepsize. We have seen that these results agree with the theoretical aspects. This method can be extended to solve regular Sturm-Liouville eigenvalue problems with Robin (mixed) boundary conditions and to some nonlinear eigenvalue problems to investigate the behavior of the eigenvalues and eigenfunction. However, this method is best suited to find small eigenvalues for the other nonlinear problems in literature. One possible method of improving its efficiency for higher eigenvalues may be to follow the ideas of $[10,12,13]$ and for eigenvalue problems for partial differential equations given in elsewhere [14-18].

\section{References}

[1] J. D. Pryce, Numerical Solution of Sturm-Liouville Problems, Monographs on Numerical Analysis, The Clarendon Press Oxford University Press, New York, NY, USA, 1993.

[2] I. Stakgold, "Branching of solutions of nonlinear equations," SIAM Review, vol. 13, pp. 289-332, 1971.

[3] R. M. Jones, Buckling of Bars, Plates, and Shells, Bull Ridge, Virginia, Va, USA, 2006.

[4] G. Domokos and P. Holmes, "Euler's problem, Euler's method, and the standard map; or, The discrete charm of buckling," Journal of Nonlinear Science, vol. 3, no. 1, pp. 109-151, 1993.

[5] D. H. Griffel, Applied Functional Analysis, Ellis Horwood Series in Mathematics and Its Applications, Ellis Horwood, Chichester, UK, 1981.

[6] L. Euler, "Methodus inveniendi lineas curvas maximi minimive proprietate gaudentes ostwald's klassiker der exakten wiss," Laussane and Geneva, vol. 75, 1774.

[7] M. E. Adiyaman and S. Somali, "Taylor's decomposition on two points for one-dimensional Bratu problem," Numerical Methods for Partial Differential Equations, vol. 26, no. 2, pp. 412-425, 2010.

[8] A. Ashyralyev and P. E. Sobolevskii, New difference schemes for partial differential equations, vol. 148 of Operator Theory: Advances and Applications, Birkhäuser, Basel, Switzerland, 2004.

[9] N. M. Bujurke, C. S. Salimath, and S. C. Shiralashetti, "Computation of eigenvalues and solutions of regular Sturm-Liouville problems using Haar wavelets," Journal of Computational and Applied Mathematics, vol. 219, no. 1, pp. 90-101, 2008.

[10] A. L. Andrew, "Asymptotic correction of Numerov's eigenvalue estimates with natural boundary conditions," Journal of Computational and Applied Mathematics, vol. 125, no. 1-2, pp. 359-366, 2000.

[11] B. van Brunt, The Calculus of Variations, Universitext, Springer, New York, NY, USA, 2004.

[12] R. S. Anderssen and F. R. de Hoog, "On the correction of finite difference eigenvalue approximations for Sturm-Liouville problems with general boundary conditions," BIT Numerical Mathematics, vol. 24, no. 4, pp. 401-412, 1984.

[13] S. Somali and V. Oger, "Improvement of eigenvalues of Sturm-Liouville problem with $t$-periodic boundary conditions," Journal of Computational and Applied Mathematics, vol. 180, no. 2, pp. 433-441, 2005.

[14] V. Mehrmann and A. Miedlar, “Adaptive computation of smallest eigenvalues of self-adjoint elliptic partial differential equations," Numerical Linear Algebra with Applications, vol. 18, no. 3, pp. 387-409, 2011.

[15] Q.-M. Cheng, T. Ichikawa, and S. Mametsuka, "Estimates for eigenvalues of the poly-Laplacian with any order in a unit sphere," Calculus of Variations and Partial Differential Equations, vol. 36, no. 4, pp. 507-523, 2009.

[16] C. Lovadina, M. Lyly, and R. Stenberg, "A posteriori estimates for the Stokes eigenvalue problem," Numerical Methods for Partial Differential Equations, vol. 25, no. 1, pp. 244-257, 2009.

[17] S. Jia, H. Xie, X. Yin, and S. Gao, "Approximation and eigenvalue extrapolation of biharmonic eigenvalue problem by nonconforming finite element methods," Numerical Methods for Partial Differential Equations, vol. 24, no. 2, pp. 435-448, 2008.

[18] C. V. Verhoosel, M. A. Gutiérrez, and S. J. Hulshoff, "Iterative solution of the random eigenvalue problem with application to spectral stochastic finite element systems," International Journal for Numerical Methods in Engineering, vol. 68, no. 4, pp. 401-424, 2006. 


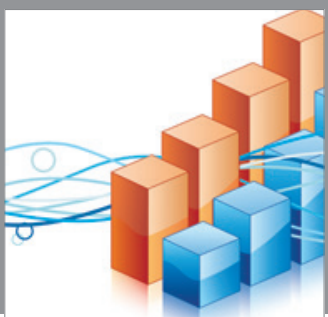

Advances in

Operations Research

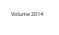

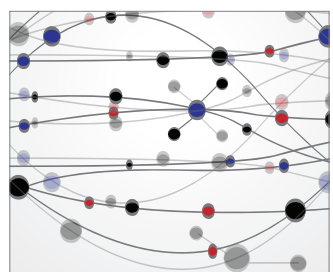

\section{The Scientific} World Journal
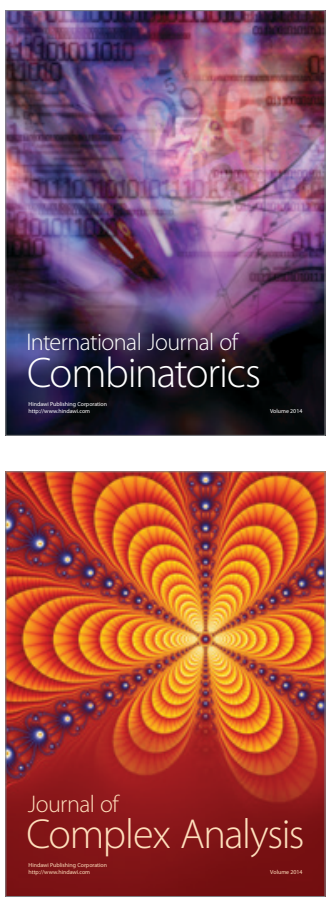

International Journal of

Mathematics and

Mathematical

Sciences
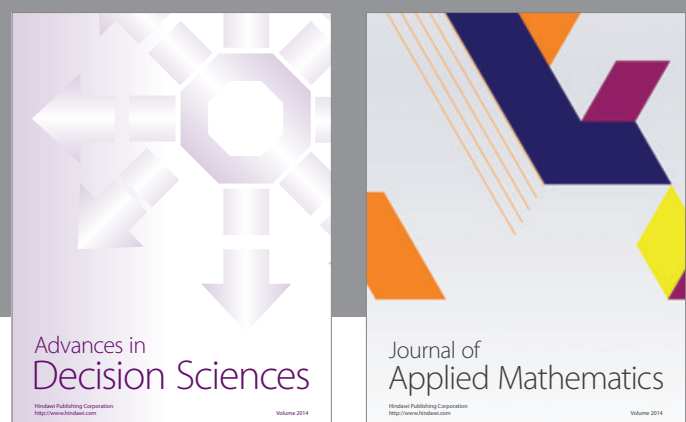

Journal of

Applied Mathematics
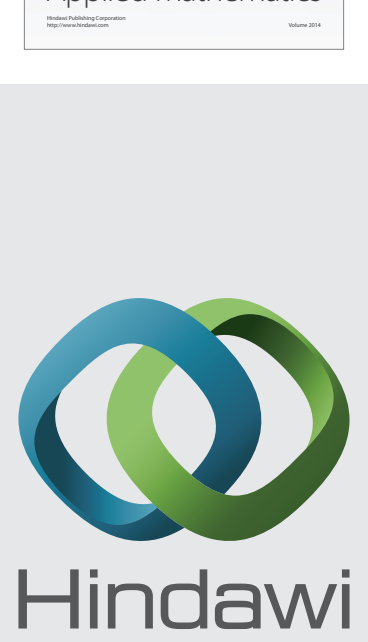

Submit your manuscripts at http://www.hindawi.com
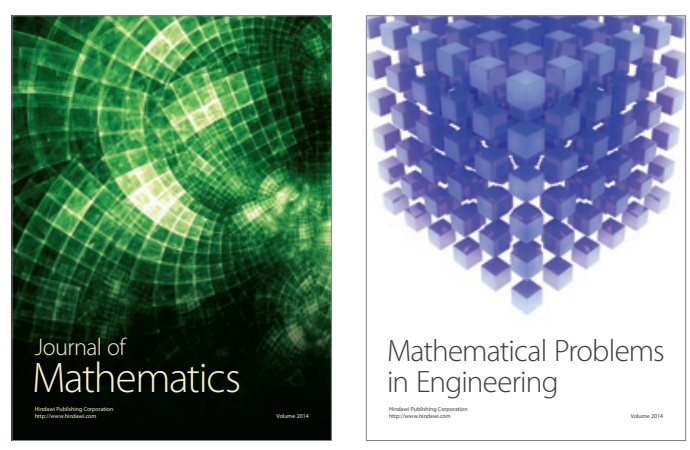

Mathematical Problems in Engineering
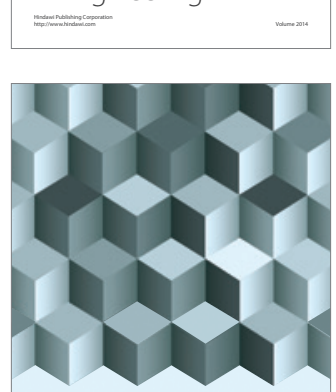

Journal of

Function Spaces
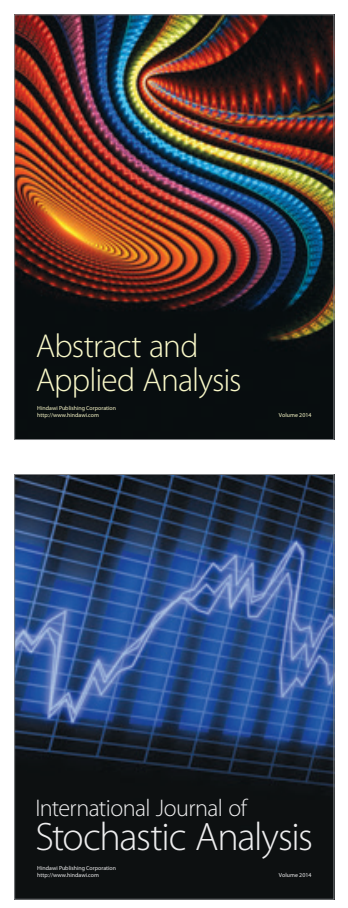

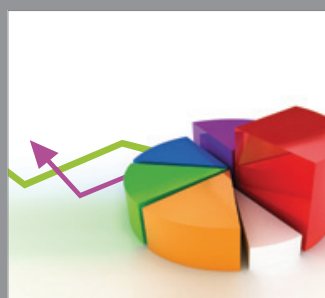

ournal of

Probability and Statistics

Promensencen
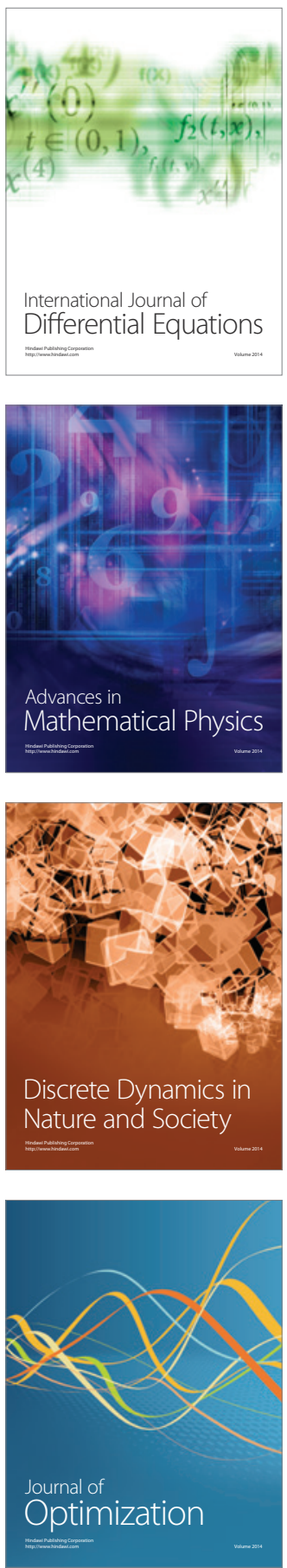